ORIGINAL ARTICLE

\title{
Early prediction of individual outcome following cardiopulmonary resuscitation: systematic review
}

\author{
P Kaye
}

Emerg Med J 2005;22:700-705. doi: 10.1136/emj.2004.016253

Correspondence to: P Kaye, Frenchay Hospital, Bristol, UK; philip_bath@ hotmail.com

Accepted for publication 30 September 2004
Following resuscitation from cardiorespiratory arrest $80 \%$ of patients are comatose. Of these patients, $20 \%$ will survive and regain consciousness. Is it possible to predict an individual's long term outcome at presentation and alter management accordingly? This review examines the current medical literature and demonstrates it is impossible to predict immediately outcome from hypoxic-ischaemic coma except in a small subgroup of patients with poor premorbid factors. As individual prognosis cannot be determined in the emergency department all patients who do not have significant premorbid features should proceed to a period of supportive care in the intensive care unit. Therapeutic hypothermia should be considered for these patients.
A fter a cardiopulmonary arrest and return of spontaneous circulation (ROSC), $80 \%$ of patients are comatose for a varying period of time. ${ }^{1}$ A considerable number of these remain unconscious due to hypoxicischaemic cerebral dysfunction and progress to brain death or persistent vegetative state. However $20 \%$ will survive and regain consciousness. ${ }^{2}$ As meaningful neurological recovery only occurs in a small proportion of patients and treatment is complex, expensive, possibly prolonged, and very difficult for relatives it would be helpful to be able to prognosticate on individual patients from an early stage. Many approaches have been examined to try to predict the outcome of post cardiopulmonary resuscitation (CPR) coma. These include:

- Premorbid, peri-arrest, and immediate post-arrest variables

- Serial neurological examinations looking for normal or abnormal signs or both

- Electrophysiological investigations

- Neuroimaging

- Neurobiochemical investigations

The purpose of this review is to determine the appropriate emergency management of patients resuscitated to a comatose state following cardiorespiratory arrest.

\section{REVIEW METHODS}

The Medline database (1996-2003) was searched using the Ovid search engine. MeSH headings of "Cardiorespiratory arrest survival", "Cardiorespiratory arrest outcome", Cardiac arrest survival" and "Cardiac arrest outcome" were used. These were combined with those from the MeSH headings "Coma", "Neurological outcome" and "Encephalopathy". The search was restricted to "humans", "adults" and "English language". A total of 136 papers were identified of which 25 were directly relevant to at least a part of the review question. All of the references in the chosen papers were examined to identify other relevant referenced papers. The EMBASE database and the Cochrane Library Issue 3, 2004, were also searched but provided no additional data. The journals Resuscitation and Critical Care Medicine were also handsearched from 1998 to 2003.

\section{DISCUSSION}

Premorbid, peri-arrest, and immediate post-arrest variables

Premorbid, peri-arrest, and post-arrest variables have been extensively examined, largely in retrospective analyses. Most studies have analysed survival rather than neurological outcome.

\section{Premorbid factors}

Age

Schultz et al reported a retrospective review of 75 post-arrest patients and showed a significant difference in survival between patients under the age of 60 years and those over the age of 80 years $\left(15 \% \vee 4 \%\right.$, respectively). ${ }^{3}$ They did not examine neurological outcome. However, Berger and Kelley in a prospective analysis of 255 inhospital cardiopulmonary arrests in non-critical patients demonstrated age was not an independent predictor of survival. ${ }^{4}$ Varon and kelley reported substantial survival among the elderly after an inhospital arrest with eight survivors from 89 patients (11\%) over the age of 70 years. ${ }^{5}$ Parish et al reported, in a retrospective review of 2813 cardiorespiratory arrests, that age was unrelated to survival where the initial rhythm was pulseless ventricular tachycardia (VT), ventricular fibrillation (VF), or asystole, but it was related if the rhythm was pulseless electrical activity (PEA). The latter was thought to reflect the poor premorbid state associated with a PEA arrest. ${ }^{6}$ Importantly Rogove et al reported in a prospective study of 774 patients that old age did not negate good cerebral outcome after cardiorespiratory arrest. $^{7}$

\section{Premorbid health and performance status}

Berger and Kelley ${ }^{4}$ reported that patients' admission diagnoses and comorbidities predicted outcome from inhospital cardiorespiratory arrest. This is reflected in the increased mortality rate from a PEA arrest, a common endpoint of severe illness. Three morbidity scores have been developed as a guide to "Do Not Attempt Resuscitation" (DNAR) decisions but the information they provide is relevant to prognosis after an arrest. The Prognosis after Resuscitation (PAR) score was developed from a meta-analysis of 14 post-arrest studies in $1992^{8}$ and uses seven variables: cancer, sepsis, poor performance status, pneumonia, creatinine $>130 \mathrm{mg} / \mathrm{l}$, and age over 70 as positive scores, and recent myocardial infarction as a negative score. Prospective validation in 274 consecutive

\footnotetext{
Abbreviations: CPR, cardiopulmonary resuscitation; $\mathrm{CK}$, creatine kinase; DNAR, Do Not Attempt Resuscitation; EEG, electroencephalography; NSE, neurone-specific enolase; PAR, Prognosis after Resuscitation; PEA, pulseless electrical activity; ROSC, return of spontaneous circulation; (S)SEP, (somato)sensory evoked potential; VF, ventricular fibrillation; VT, ventricular tachycardia
} 
resuscitated patients demonstrated that a score of $>4$ predicted non-survival. Bowker and Stewart ${ }^{9}$ compared the PAR to other morbidity scoring systems in a group of elderly patients and showed that they were all more complex to use due to multiple variables, in one case the score had not been validated prospectively, and they provided no additional information. Unfortunately although $100 \%$ specific and therefore of use in DNAR decisions, the PAR score does not help in the majority of post-arrest patients who will have low scores. It also does not predict a good neurological outcome in those predicted to survive.

\section{Peri-arrest factors}

Schultz et $a l^{3}$ reported that the duration of the cardiorespiratory arrest was related to outcome. They reported survival rates of $48 \%$ for less than 10 minutes duration and $2 \%$ for longer than 10 minutes. Other studies have confirmed this highly biologically plausible finding using surrogate markers for duration of arrest. Behringer et al reported an unfavourable cerebral performance category was associated with significantly greater cumulative doses of adrenaline (epinephrine). ${ }^{10}$ This was confirmed by Denton and Thomas who also correlated the number of DC shocks with poor outcome. ${ }^{11}$ Saklayen et al ${ }^{12}$ confirmed a shorter duration of arrest was associated with a better outcome and that this correlated with a witnessed arrest or resuscitation by a health professional indicating earlier effective intervention. They also demonstrated that pulseless VT or VF arrests had a better outcome than PEA arrests, and that arrests in the emergency department or coronary care unit had a better outcome than those in intensive care unit (ITU) or a general ward. The latter finding reflects both the arrest rhythm and the premorbid state of the patients. Andreasson et al quantified these observations in cardiorespiratory arrests in hospital. ${ }^{13}$ They showed a survival rate of $64 \%$ from VT/VF arrest, $24 \%$ from asystole, and $10 \%$ from a PEA arrest. Monitored patients had a survival rate of 52\% while unmonitored patients had a survival rate of $27 \%$.

\section{Post-arrest factors}

Various post-arrest physiological variables have been investigated as proposed prognostic indicators post cardiorespiratory arrest with ROSC. Schultz et al examined initial $\mathrm{PaO}_{2}$ after ROSC and showed that a level of $<50 \mathrm{~mm} \mathrm{Hg}$ correlated with survival of $1 \%$ compared with $13 \%$ with a level $>50 \mathrm{~mm} \mathrm{Hg}$. APACHE II scores were shown to correlate with outcome by Denton and Thomas. ${ }^{11}$ However, Berger and Kelley $^{4}$ showed that although APACHE II scores correlated with ITU survival, there was no correlation with hospital discharge. Niskanen et al, using an APACHE II score of 25 or greater as a cut-off, showed a correlation with poor outcome but the positive predictive value was only $71 \% .^{14}$

Arterial blood gas analysis after ROSC has also been investigated. Schultz et $a l^{3}$ showed no correlation between initial $\mathrm{pH}$ and survival. Denton and Thomas ${ }^{11}$ showed correlation between arterial bicarbonate concentration on the first blood gas analysis in ITU and outcome but showed no correlation with $\mathrm{pH}$ of the same sample or initial bicarbonate level following ROSC. Mullner et $a^{15}$ showed a trend for higher levels of serum lactate after ROSC to correlate with poor neurological outcome but a level of greater than $16 \mathrm{mmol} / \mathrm{l}$ was required for $100 \%$ specificity. Buunk et al $^{16}$ examined oxygen delivery following ROSC as a prognostic indicator by comparing mixed venous $\left(\mathrm{SmvO}_{2}\right)$ and jugular bulb venous $\left(\mathrm{SjO}_{2}\right)$ oxygen saturation. Post-arrest $\mathrm{SjO}_{2}$ was about $10 \%$ lower than the $\mathrm{SmvO}_{2}$. In non-survivors $\mathrm{SjO}_{2}$ steadily increased due to reduced cerebral oxygen consumption secondary to loss of functional tissue. At 24 hours an $\mathrm{Sjo}_{2}$ greater than $\mathrm{Smvo}_{2}$ had a positive predictive value for poor outcome of $93 \%$. Denton and Thomas ${ }^{11}$ also demonstrated that an inotrope requirement after ROSC correlated with a poor outcome. This was confirmed by Langhelle et $a l^{17}$ in a retrospective cohort analysis of 459 patients who had an out of hospital cardiorespiratory arrest. They demonstrated that hypotension, oliguria, base deficit of 3.5 or more, plasma glucose of greater than $10.6 \mathrm{mmol} / \mathrm{l}$, or core body temperature of $>37.8{ }^{\circ} \mathrm{C}$ within 24 hours of ROSC were each independently related to poor outcome. The latter two factors are consistent with other studies of outcome from hypoxic-ischaemic coma. These have demonstrated that hyperglycaemia following an arrest has a statistically significant impact on the risk of poor neurological outcome whereas for every degree Celsius above $37{ }^{\circ} \mathrm{C}$ the risk of an unfavourable neurological outcome increases with an odds ratio of $2.26 . .^{18} 19$

\section{Serial neurological examination \\ Abnormal neurological signs}

Myoclonus in comatose patients following ROSC was reported to be an agonal sign by Wijdicks et al as all patients with myoclonus died. ${ }^{20}$ However, since that report this finding has been contradicted in a number of case reports. Morris et $a l^{21}$ reported three survivors with mild disability and in a literature review found five similar cases. Snyder et $a^{22}$ reported seizure activity in $30 \%$ of patients following ROSC; $17 \%$ of those with myoclonus, $33 \%$ of those with partial seizures, and $50 \%$ of those with generalised, complex seizures survived. Overall the survival rate for those with seizure activity was $32 \%$ compared with $43 \%$ for those with no seizure activity. A further report by Krumholz et al ${ }^{23}$ showed no relation between seizure activity and neurological outcome, except in a subgroup with status, which predicted a higher risk of persistent unconsciousness or brain death.

Jorgenson and Holm examined neurological outcome over one year in 231 patients post cardiorespiratory arrest with ROSC. ${ }^{24}$ They divided outcome into four categories: brain death, persistent unconsciousness, persistent disability after awakening and complete recovery. They reviewed both electroencephalographic (EEG) activity (see later) and the presence of abnormal neurological signs in an attempt to predict individual neurological outcome. Brain stem areflexia with apnoea (brain death) noted at any time after ROSC predicted brain death with $100 \%$ specificity and sensitivity. Myoclonus was unhelpful in prediction occurring in 65 patients with brain death or persistent unconsciousness and 27 with persistent disability after awakening or complete recovery. Adding the presence of myoclonus at any time to the presence of no EEG activity at 1 hour after ROSC only marginally improved the predictive value-that is, 53 with brain death or persistent unconsciousness versus 13 with persistent disability after awakening or complete recovery. A systematic review in 1998 combining 33 studies of prognostic indicators post-arrest with ROSC demonstrated the poor predictive value of seizure activity. Specificity and sensitivity for poor outcome were $25-92 \%$ and $16-85 \%$, respectively..$^{25}$

\section{Glasgow Coma Score}

The Glasgow Coma Scale (GCS) has been extensively investigated as a predictor of individual outcome following cardiac arrest with ROSC. Zandbergen et al in a systematic review showed that a GCS of 5 or less in the first 24 hours was not helpful in predicting outcome. ${ }^{25}$ Mullie et al in a study of 133 patients showed that of 54 patients with a GCS of 4 or less 48 hours after ROSC only one recovered consciousness. A GCS of 10 or more at the same time predicted good recovery in 40 of 49 patients. ${ }^{26}$ Bassetti et al combined a GCS of 8 or less with abnormal somatosensory evoked potentials at 48 hours to produce a $97 \%$ specificity for 
brain death or persistent unconsciousness. ${ }^{27}$ Grubb et a ${ }^{28}$ showed that a GCS of 8 or less was absolutely predictive of poor outcome at 72 hours. However, this study included small numbers of patients in this category. A systematic review in $2004^{29}$ of clinical signs in prognostication following cardiac arrest with ROSC demonstrated that an absent motor response at 72 hours had a likelihood ratio for death or poor neurological outcome of 9.2 (95\% CI 2.1 to 49.4). This indicates it is impossible to use a low GCS as an absolute predictor of poor outcome though it suggests a low probability of a good outcome. It should be noted that a significant limitation in all prognostication studies is that in many cases active support has been withdrawn as soon as the patient appears to demonstrate poor neurological recovery and this may bias results in favour of a poor outcome.

\section{Temporal recovery of normal cerebral function}

Persistent brainstem dysfunction is an indicator of poor prognosis post arrest with ROSC as the adult cerebral cortex is more susceptible to the effects of anoxia than the brainstem. ${ }^{26}$ In view of this brainstem reflex activity has been examined as a predictor of individual outcome. The simplest clinical examination is the pupillary response to light. Numerous studies have raised doubts about the specificity of pupillary responsivity due to small numbers of patients who make a good recovery despite no response to light. Longstreth et al, for example, reported 4 out of 39 patients with absent pupillary responses had a good outcome. ${ }^{30}$ However in these studies there is usually a failure to examine the temporal relation between the time of testing and final outcome. Zandbergen et al in a systematic review in $1998^{25}$ reported three independent factors with $100 \%$ specificity for poor outcome. These were absence of pupillary response to light on day 3 , absent motor response to pain on day 3, and abnormal evoked potential tests within one week (see below).

Jorgenson $^{31}$ examined the natural course of neurological recovery following CPR and demonstrated that although the magnitude and duration of the hypoxic-ischaemic cerebral insult prior to ROSC determined if cerebral function could be restored, in all cases where neurological recovery occurred, it occurred in the same sequence. Brain stem functions recovered in the same sequential order irrespective of initial neurological status or eventual outcome and this recovery of function followed a specific temporal course. The initial phase was a phase of exclusive presence of cranial nerve reflexes. Stagnation of recovery in terms of lack of timerelated recovery of function predicted either brain death or persistent unconsciousness. Stagnation of recovery did not occur in those with persistent disability after awakening or complete recovery and these findings did not aid in prediction of final outcome between these groups. The study determined the recovery sequence as follows: initially recovery of spontaneous ventilation, then pupillary light response, then coughing/swallowing then presence of a ciliospinal reflex. It also examined critical time limits for neurological recovery. Pupillary responsivity to light at two minutes following ROSC had a positive predictive value of 1.0 for persistent disability after awakening or complete recovery whereas absence of responsivity at 20 minutes after ROSC had a negative predictive value of 0.98 . Absence of a cough/ swallow response at 30 minutes after ROSC had a negative predictive value for persistent disability after awakening or complete recovery of 1.0. Presence of a ciliospinal reflex at 20 minutes following ROSC had a positive predictive value of 1.0 for persistent disability after awakening or complete recovery and absence at 30 minutes after ROSC gave a negative predictive value of 0.98 .
Further work by Jorgensen and Holm examined additional potential unique discriminative signs in 231 patients following arrest with ROSC. ${ }^{24}$ They demonstrated that absence of the caloric vestibular reflex at eight hours after ROSC had a negative predictive value of 1.0 for complete recovery or persistent disability after awakening. They also demonstrated that by using a combination of early EEG with division of patients into those with no activity versus some activity at one hour after ROSC they could use the time of recovery of speech in each group to differentiate persistent disability after awakening and complete recovery. For example, speech at 24 hours in those with some EEG activity at one hour has a positive predictive value for complete recovery of 1.0.

\section{Electrophysiological investigations Sensory evoked potentials}

Sensory (especially somatosensory) evoked potentials (SEP) have been investigated for their potential in prediction of outcome from coma for over 20 years. $^{32}$ They have the advantage of being a non-invasive bedside test with high reproducibility. They are less susceptible to electrical interference $^{33}$ and are less affected by sedation or the presence of a septic or metabolic encephalopathy ${ }^{34}$ than EEGs.

Madl et al ${ }^{35}$ published a study in 1993 of 66 patients investigated with SSEP between 4 and 48 hours after ROSC. In 17 patients with "favourable outcome" a normal response was demonstrated whereas in 49 with a "poor outcome" the evoked response was delayed or absent. However, further studies have qualified these initial findings. A study of SEP in 62 patients within 24 hours of $\operatorname{ROSC}^{34}$ demonstrated an abnormal SEP was associated with a "poor prognosis" but a normal SEP did not predict recovery. Nakabayashi et al, ${ }^{33}$ Chen et $a l^{34}$ and Sandroni et $a l^{36}$ demonstrated a $100 \%$ negative predictive value for a good outcome (persistent disability after awakening or complete recovery) with delayed or absent SEPs but a poor positive predictive value for normal SEPs. Nakabayashi demonstrated that of 12 patients with normal cortical response on SSEP, eight recovered consciousness. Chen demonstrated that bilaterally absent or low amplitude SSEP predicted brain death or persistent unconsciousness while with a normal SSEP the rate of complete recovery was only $44 \%$. This lack of sensitivity was confirmed in a systematic review in $1998 .{ }^{25}$ This showed that bilateral absence of early cortical response to SEP within the first week had a positive likelihood ratio of poor outcome of 12 (CI 5.3 to 27.2 ). Specificity was $100 \%$, but sensitivity was $28-73 \%$.

Gendo et al recorded serial SEPs at 4, 12, 24, and 48 hours after ROSC. $^{37}$ They demonstrated that SEPs improved significantly between 4 and 24 hours with no improvement afterwards. Newer studies of SEPs following ROSC have tended to adopt a policy of investigation at 24 hours in view of these findings.

It has been suggested that as the somatosensory cortex is relatively resistant to hypoxic-ischaemic injury it may be preserved even when other cortical areas are severely affected. This would explain why absence/delay of SEP reinforces the likelihood of poor outcome whereas their preservation does not necessarily predict a favourable outcome. ${ }^{34}$

\section{Electroencephalography}

EEG studies have been used to try to predict individual outcome from coma following cardiac arrest. There have been few studies examining temporal changes in EEG making single examinations difficult to interpret. EEG patterns can also be affected by confounding factors such as electrical interference, sedative medications, and septic or metabolic encephalopathies. ${ }^{25}$ 
Synek ${ }^{38}$ produced a five point grading system in 1990 for EEG at 24 hours after ROSC and reported a 98.4\% prognostic accuracy. Yamashita et $a^{l^{39}}$ in a prospective study of 79 patients 24 hours following ROSC used a similar scale. They reported that grades I and II made a full recovery and grades IV and V were characterised by persistent unconsciousness or brain death. However, Rothstein ${ }^{40}$ in a prospective study of 40 patients demonstrated that a "malignant EEG pattern" within 48 hours of ROSC predicted a poor outcome (persistent unconsciousness or brain death) in only $42 \%$ of cases. Jorgensen and Holm took EEGs of 231 patients one hour following ROSC. In patients with no EEG activity, brain death or persistent unconsciousness was the final outcome in $68 \%$ compared with $28 \%$ in those with some EEG activity. However, although a good outcome was less likely with no EEG activity, $12 \%$ of such patients made a complete recovery. They concluded that recovery following arrest with ROSC is dictated by the severity of the hypoxic-ischaemic cerebral insult as reflected by the EEG evidence of cortical activity. However, the course and rate of recovery must be influenced by other factors as the entire spectrum of outcomes is noted regardless of initial EEG status. ${ }^{24}$

A systematic review in $1998^{25}$ suggested that a burst suppression or isoelectric pattern on EEG within the first week predicted poor outcome (persistent unconsciousness or brain death). The positive likelihood ratio of poor outcome was 9.0 (CI 2.5 to 33.1 ).

\section{Neuroimaging}

There are a number of case reports and small retrospective reviews of neuroimaging after cardiac arrest with ROSC. Computed tomography (CT), magnetic resonance imaging and positron emission tomography have all demonstrated loss of distinction between grey and white matter, developing cerebral atrophy, and hypodensities in the basal ganglia consistent with the high metabolic requirements of the cerebral cortex and basal ganglia.$^{41}$ Few studies have tried to use neuroimaging to predict outcome in comatose patients. Torbey et al carried out a retrospective review of 25 patients who had CT of the brain within 24 hours of ROSC..$^{42}$ They showed that loss of distinction between grey and white matter on CT predicted poor outcome especially at the basal ganglia level and produced a qualitative analysis that suggested a cut-off for loss of distinction and guaranteed poor outcome. This was based on a small sample and has yet to be validated..$^{42}$

\section{Neurobiochemical investigations}

Biochemical markers have been investigated in coma patients following ROSC. Their utility in possible prediction of outcome is based on the fact that they reflect cerebral structural damage rather than function. This suggests the possibility of correlating their level directly with outcome measures.

\section{Cerebrospinal fluid creatine kinase-BB isoenzyme}

The brain is rich in creatine kinase $(\mathrm{CK})$ - $\mathrm{BB}$ isoenzyme and has no CK-MM or CK-MB activity. CK-BB activity is unaffected by confounding factors that can reversibly affect cerebral function and reflects structural injury. Animal and human studies have demonstrated a correlation between cerebrospinal fluid (CSF) CK-BB after arrest and ROSC and the degree of brain injury. ${ }^{43}$ The peak CK-BB level occurs 48 72 hours after ROSC. ${ }^{44}$ Tirschwell et al tested CSF CK-BB 4872 hours after ROSC in 351 patients. They reported a sensitivity of 0.82 , a specificity of 0.85 , and a positive predictive value of 0.96 for never awakening for CK-BB levels greater than $50 \mathrm{IU} / \mathrm{l}$. A higher CK-BB cut-off of $205 \mathrm{IU} / \mathrm{l}$ increased specificity to $100 \%$ and positive predictive value to 1.0 with a reduced sensitivity of $48 \%{ }^{45}$

\section{Serum neurone-specific enolase}

Serum neurone-specific enolase (NSE) levels reflect structural cerebral damage. A small study in 43 patients with daily serum levels for seven days post arrest with ROSC demonstrated that an NSE level greater than $33 \mathrm{ng} / \mathrm{ml}$ predicts persistent unconsciousness or brain death. The specificity was $100 \%$ with a sensitivity of $80 \%$. Unfortunately the temporal relationship was unclear. ${ }^{46}$ Other small studies have confirmed a correlation between NSE levels and outcome. Zingler et al measured serial NSE levels after ROSC and demonstrated that an NSE level of greater than $43 \mathrm{ng} / \mathrm{ml}$ on day 2 had a specificity of $100 \%$ and a sensitivity of $91 \%$ for a poor outcome. ${ }^{47}$

\section{Serum astroglial protein S-100}

Serum S-100 levels reflect structural cerebral damage. Rosen et $a l^{48}$ in a study of $4 \mathrm{l}$ patients post out of hospital cardiac arrest demonstrated a significantly higher level of S-100 in non-survivors. An S-100 level of $0.2 \mathrm{ng} / \mathrm{ml}$ or greater on day 2 had a positive predictive value of $100 \%$ for death within 14 days whereas a level of less than 0.2 had a positive predictive value for survival of $89 \%$. Bottiger et $a l^{49}$ in a study of 66 patients post out of hospital cardiac arrest demonstrated that S-100 levels two hours following ROSC were significantly higher in patients with brain damage (including survivors) than in those who recovered with no brain damage.

A systematic review of the predictive utility of biochemical markers of brain damage in 2001 concluded that although the results for CK-BB were interesting and produced no false positive results the small numbers and poor methodology in all trials made the combined results for any marker insufficiently accurate to provide a solid basis for nontreatment decisions. ${ }^{50}$

\section{THERAPEUTIC HYPOTHERMIA}

The management of hypoxic-ischaemic coma after a cardiorespiratory arrest should now include induced mild hypothermia. An advisory statement from the International Liaison Committee on Resuscitation in 2003 has recommended cooling to $32-34{ }^{\circ} \mathrm{C}$ for $12-24$ hours for all patients after ROSC following out of hospital cardiac arrest where the initial rhythm was VF. The statement also recommends consideration in other rhythms and inhospital arrests. ${ }^{51}$ This advice is largely based on two prospective randomised studies which reported numbers needed to treat for a favourable neurological outcome at six months as $6^{52}$ and $4 .^{53}$ It is unclear how therapeutic hypothermia will affect the normal temporal sequence of cerebral recovery. Importantly the interval between ROSC and attainment of the required core temperature had an interquartile range of 4-16 hours in one study. ${ }^{52}$ Examination will however be compromised by the associated requirement for sedation and paralysis.

\section{CONCLUSION}

A wide range of investigations has been utilised to try to predict individual outcome from coma due to hypoxicischaemic encephalopathy after cardiorespiratory arrest with ROSC.

- Age is not a significant factor in outcome provided the arrest is not an endstage event as indicated by a PEA rhythm.

- Comorbidity and premorbid performance status are good individual predictors of poor outcome following cardiorespiratory arrest and ROSC. However they can only be 


\section{Bottom line}

- It is impossible to predict immediately outcome from hypoxic-ischaemic coma following ROSC except in a small subgroup of patients with poor premorbid factors indicating a poor physiological reserve.

- Very early prediction of poor outcome may be possible where features of brain death are present or where early recovery of specific cranial nerve reflexes fails to occur. However this temporal sequence may be altered by therapeutic hypothermia.

- All patients who remain comatose after ROSC and who do not have significant pre-morbid features should progress to a period of supportive care in the ITU. Therapeutic hypothermia, usually requiring sedation and paralysis, should be considered.

- Individual prognosis cannot be determined in the emergency department.

used to identify a small subgroup with poor outcome and do not predict awakening in survivors.

- Prolonged resuscitation (or surrogate markers for prolonged resuscitation) is associated with poor outcome as would be expected with hypoxic-ischaemic pathology. However it is impossible to absolutely predict poor outcome from arrest duration alone once ROSC is achieved.

- Arrest rhythms reflect the patient's premorbid state and predict outcome but provide no individual prognostic evidence. The better survival from emergency department or coronary care unit arrests is a function of early intervention and an increased probability of a reversible cause but once ROSC is achieved, again individual prognosis is difficult to assess if the patient is comatose.

- Measured physiological variables demonstrate that the worse the post-arrest milieu the more likely there is to be a poor neurological outcome but none are sufficiently sensitive indicators to predict outcome.

- Abnormal neurological signs are unhelpful in prediction of outcome and may simply reflect the normal course of cerebral recovery following a hypoxic-ischaemic insult. Only features consistent with brain death at any time provide an absolute prediction for brain death.

- A GCS score of 8 or less or an absent motor response at 72 hours are suggestive of poor outcome but do not provide an absolute prognostic prediction.

- Serial neurological examination is the most useful means of predicting individual outcome following ROSC. The course of neurological recovery from hypoxic-ischaemic coma has been shown to be fixed and sequential within an established time frame. Failure of this sequence to progress, delay or stagnation of recovery, or loss of initially recovered function predict failure of awakening. The short and early time frame for the first phase of recovery (the phase of exclusive presence of cranial nerve reflexes) allows very early prognostic decisions to be made.

- SEPs have shown some utility and are unaffected by confounding factors. Abnormalities are $100 \%$ specific for poor outcome though only after 24 hours of coma. The converse does not predict good outcome. The availability of SEP investigation is limited.

- EEG examination is insufficiently sensitive or specific to provide any prognostic certainty especially as numerous confounding factors have been identified.
- Neuroimaging after ROSC has not been studied extensively and at present is unhelpful in prognostication.

- Neurobiochemical markers reflect structural cerebral damage and therefore should predict neurological outcome rather than reflect current function. However their individual predictive value is variable and is unhelpful before 48 hours have elapsed. At present they are at best adjuncts to assessment of neurological outcome.

Competing interests: none declared

\section{REFERENCES}

1 Madl C, Holzer M. Brain function after resuscitation from cardiac arrest. Curr Opin Crit Care 2004;10:213-17.

2 Zandbergen EG, de Haan RJ, Reitsma JB, et al. Survival and recovery of consciousness in anoxic-ischaemic coma after cardiopulmonary resuscitation. Intensive Care Med 2003;29:1911-15.

3 Schultz SC, Cullinane DC, Pasquale MD, et al. Predicting in-hospital mortality during cardiopulmonary resuscitation. Resuscitation 1996;33:13-17.

4 Berger R, Kelley M. Survival after in-hospital cardiopulmonary arrest of noncritically ill patients. A prospective study. Chest 1994;106:872-79.

5 Varon J, Fromm RE. In hospital resuscitation among the elderly: substantial survival to hospital discharge. Am J Emerg Med 1996;14:130-2.

6 Parish DC, Dane DC, Montgomery M, et al. Resuscitation in the hospital: differential relationships between age and survival across rhythms. Crit Care Med 1999;10:2137-41.

7 Rogove HJ, Safar P, Sutton-Tyrrell K, et al. Old age does not negate good cerebral outcome after cardiopulmonary resuscitation: analyses from the brain resuscitation clinical trials. The Brain Resuscitation Clinical Trial I and II Study Groups. Crit Care Med 1995;23:18-25.

8 Ebell MH. Prearrest predictors of survival following in hospital cardiopulmonary resuscitation; a metanalysis. J Fam Pract 1992;34:551-8. 9 Bowker L, Stewart K. Predicting unsuccessful cardiopulmonary resuscitation (CPR): a comparison of three morbidity scores. Resuscitation 1999;40:89-95.

10 Behringer W, Kittler H, Sterz F, et al. Cumulative epinephrine dose during cardiopulmonary resuscitation and neurological outcome. Ann Intern Med 1998; 129:450-6.

11 Denton R, Thomas AN. Cardiopulmonary arrest: a retrospective review. Anaesthesia 1997;52:137-40.

12 Saklayen M, Liss H, Markert R. In-hospital cardiopulmonary resuscitation Survival in 1 hospital and literature review. Medicine 1995;74:163-75.

13 Andreasson AC, Herlitz J, Bang A, et al. Characteristics and outcome among patients with a suspected in-hospital cardiac arrest. Resuscitation 1998;39:23-31.

14 Niskanen M, Kari A, Nikki P, et al. Acute physiology and chronic health evaluation (APACHE II) scores and Glasgow coma scores as predictors of cardiac arrest. Crit Care Med 1991;19:1465-73.

15 Mullner M, Sterz F, Domanovits $H$, et al. The association between blood lactate concentration on admission, duration of cardiac arrest and functional neurological recovery in patients resuscitated from ventricular fibrillation. Intensive Care Med 1997;23:1138-43.

16 Buunk G, van der Hoeven JG, Meinders, et al. Prognostic significance of the difference between mixed venous and jugular bulb oxygen saturation in comatose patients resuscitated from a cardiac arrest. Resuscitation 1999;41:257-62

17 Langhelle A, Tyrold SS, Lexow K, et al. In hospital factors associated with improved outcome after out-of-hospital cardiac arrest. A comparison between 4 regions in Norway. Resuscitation 2003;56:247-63.

18 Zeiner A, Holze M, Sterz F, et al. Hyperthermia after cardiac arrest is associated with an unfavorable neurologic outcome. Arch Intern Med 2001;161:2007-12.

19 Mullner M, Sterz F, Binder M, et al. Blood glucose concentration after cardiopulmonary resuscitation influences functional neurological recovery in human cardiac arrest survivors. J Cereb Blood Flow Metab 1997;17:430-6.

20 Wijdicks EF, Parisi JE, Sharbrough FW. Prognostic value of myoclonus status in comatose survivors of cardiac arrest. Ann Neurol 1994;35:239-43.

21 Morris HR, Howard RS, Brown P. Early myoclonic status and outcome after cardiorespiratory arrest. J Neurol Neurosurg Psychiatry 1998;64:267-8.

22 Snyder BD, Hauser WA, Loewensen RB, et al. Neurological prognosis after cardiopulmonary arrest: III. Seizure activity. Neurology 1980;30:292-7.

23 Krumholz A, Stern BJ, Weiss HD. Outcome from coma after cardiopulmonary resuscitation: relation to seizures and myoclonus. Neurology 1988;38:401-5.

24 Jorgensen EO, Holm S. Prediction of neurological outcome after cardiopulmonary arrest. Resuscitation 1999;41:145-52.

25 Zandbergen EG, de Haan RJ, Stoutenbeek CP, et al. Systematic review of early prediction of poor outcome in anoxic-ischaemic coma. Lancet 1998;352:1808-13

26 Mullie A, Verstringe P, Buylaert W, et al. Predictive value of Glasgow coma score for awakening after out-of-hospital cardiac arrest. Cerebral Resuscitation Study group of the Belgian Society for Intensive Care. Lancet 1988;1:137-40.

27 Bassetti C, Bomio F, Mathis J, et al. Early prognosis in coma after cardiac arrest: a prospective clinical, electrophysiological and biochemical study of 60 patients. J Neurol Neurosurg Psychiatry 1996;61:610-15.

28 Grubb NR, Elton RA, Fox KA, et al. In-hospital mortality after out-of-hospital cardiac arrest. Lancet 1995;346:417-21. 
29 Booth CM, Boone RH, Tomlinson G, et al. Is this patient dead, vegetative or severely neurologically impaired? Assessing outcome for comatose survivors of cardiac arrest. JAMA 2004;291:870-9.

30 Longstreth WT, Diehr P, Inui TS. Prediction of awakening after out-of-hospital cardiac arrest. N Engl J Med 1983;308:1278-82.

31 Jorgensen EO. Course of neurological recovery and cerebral prognostic signs during cardiopulmonary resuscitation. Resuscitation 1997;35:9-16.

32 Ahmed I. Use of somatosensory evoked responses in the prediction of outcome from coma. Clin Electroencephalogr 1988;19:78-86.

33 Nakabayashi M, Kurokawa A, Yamamoto Y, et al. Immediate prediction of recovery of consciousness after cardiac arrest. Intensive Care Med 2001;27:1210-14.

34 Chen R, Bolton CF, Young B. Prediction of outcome in patients with anoxic coma: a clinical and electrophysiologic study. Crit Care Med 1996;24:672-78.

35 Madl C, Grimm G, Kramer L, et al. Early prediction of individual outcome after cardiopulmonary resuscitation. Lancet 1993;341:855-8.

36 Sandroni C, Barelli A, Piazza O, et al. What is the best test to predict outcome after prolonged cardiac arrest? Eur J Emerg Med 1995;2:33-7.

37 Gendo A, Kramer L, Hafner M, et al. Time-dependency of sensory evoked potentials in comatose cardiac arrest survivors. Intensive Care Med 2001;27:1305-11.

38 Synek VM. Value of a revised EEG coma scale for prognosis after cerebral anoxia and diffuse head injury. Clin Electroencephalogr 1990;21:25-30.

39 Yamashita S, Morinaga T, Ohgo S, et al. Prognostic value of electroencephalogram (EEG) in anoxic encephalopathy after cardiopulmonary resuscitation: relationship among anoxic period, EEG grading and outcome. Intern Med 1995;34:71-6.

40 Rothstein TL. Post-anoxic alpha (theta) coma: a reappraisal of its prognostic significance. Electroencephalogr Clin Neurophysiol 1991;79:101-7.

41 De Reuck J, Decoo D, Vienne J, et al. Significance of white matter lucencies in posthypoxic-ischaemic encephalopathy: comparison of clinical status and of computed and positron emission tomographic findings. Eur Neurol 1992;32:334-9.
42 Torbey MT, Selim M, Knorr J, et al. Qualitative analysis of loss of distinction between grey and white matter in comatose patients after cardiac arrest. Stroke 2000;31:2163-7.

43 Vaagenes P, Mullie A, Fodstad DT, et al. The use of cytosolic enzyme increase in cerebrospinal fluid of patients resuscitated after cardiac arrest. Brain Resuscitation Clinical Trial I Study Group. Am J Emerg Med 1994;12:621-4.

44 Mullie A, Lust P, Penninckx J, et al. Monitoring of cerebrospinal fluid enzyme levels in postischaemic encephalopathy after cardiac arrest. Crit Care Med 1981;9:399-400.

45 Tirschwell DL, Longstreth WT, Rauch-Matthews ME, et al. Cerebrospinal fluid creatine kinase $\mathrm{BB}$ isoenzyme activity and neurological prognosis after cardiac arrest. Neurology 1998;50:830-1.

46 Fogel W, Kreiger D, Veith $M$, et al. Serum neuron-specific enolase as an early predictor of outcome after cardiac arrest. Crit Care Med 1997:25:1133-8.

47 Zingler VC, Krumm B, Bertsch T, et al. Early prediction of neurological outcome after cardiopulmonary resuscitation: a multimodal approach combining neurobiochemical and electrophysiological investigations may provide higher diagnostic certainty in patients after cardiac arrest. Eur Neurol 2000:49:79-84.

48 Rosen H, Rosengren L, Herlizt J, et al. Increased serum levels of the S-100 protein are associated with hypoxic brain damage after cardiac arrest. Stroke 1998;29:473-7.

49 Bottiger BW, Mobes S, Glatzer R, et al. Astroglial protein S-100 is an early and sensitive marker of hypoxic brain damage and outcome after cardiac arrest in humans. Circulation 2001;103:2694-8.

50 Zandbergen EG, de Haan RJ, Hijdra A, et al. Systematic review of prediction of poor outcome in anoxic-ischaemic coma with biochemical markers of brain damage. Intensive Care Med 2001;27:1661-7.

51 Nolan JP, Morley PT, Hoek TL, et al. Therapeutic hypothermia after cardiac arrest. ILCOR Advisory Statement. Circulation 2003;108:118.

52 The Hypothermia after Cardiac Arrest Study Group. Mild therapeutic hypothermia to improve the neurologic outcome after cardiac arrest. N Engl J Med 2002;346:549-56.

53 Bernard SA, Gray TW, Buist MD, et al. Treatment of comatose survivors of out-of-hospital cardiac arrest with induced hypothermia. N Engl J Med 2002;346:557-63. 